\title{
Einsatz der CT-Angiographie zur Feststellung des zerebralen Zirkulationsstillstandes
}

\author{
H. Lanfermann $\cdot$ F. Götz $\cdot$ P. Raab
}

Eingegangen: 10. August 2015 / Online publiziert: 26. August 2015

(C) Die Autor(en) 2015. Dieser Artikel ist auf Springerlink.com mit Open Access verfügbar.

\begin{abstract}
Zusammenfassung Die CT-Angiographie (CTA) ist in den aktualisierten Richtlinien zur Feststellung des irreversiblen Hirnfunktionsausfalles als ergänzendes apparatives Untersuchungsverfahren zum Nachweis des zerebralen Zirkulationsstillstandes zugelassen worden. Für die Durchführung der CTA ist ein Standardprotokoll festgelegt worden. Die Auswertung der CTA muss durch Ärzte für Radiologie mit mehrjähriger neuroradiologischer Erfahrung, möglichst durch Radiologen mit dem „Schwerpunkt Neuroradiologie“ erfolgen. Hinsichtlich der Beurteilung der CTA ist das sog. ,stasis filling“, die langsam progrediente Ausbreitung des Kontrastmittels in den intrakraniellen Arterien bei sistierender zerebraler Zirkulation, zu berücksichtigen.
\end{abstract}

Schlüsselwörter Hirnfunktion · Hirntod ·

Hirndurchblutung · CT-Angiographie · „Stasis filling“ ·

Qualitätssicherung

\begin{abstract}
CT-angiography (CTA) has been accredited as an additional technical method for the detection of the cessation of cerebral blood circulation in the updated German guidelines for the determination of irreversible loss of brain function. A standardized CTA protocol was defined. The evaluation of the CTA has to be done by radiologists with several years of experience in neuroradiology, preferably by radiologists certified as neuroradiologists. The so-called "stasis filling", a slow progressive spread of contrast media into the cerebral arteries despite cessation of cerebral blood circulation, has to be considered.
\end{abstract}

H. Lanfermann $(\bowtie) \cdot$ F. Götz $\cdot$ P. Raab

Institut für Diagnostische und Interventionelle Neuroradiologie,

Medizinische Hochschule Hannover,

Carl-Neuberg-Straße 1,

30625 Hannover, Deutschland

E-Mail: lanfermann.heinrich@mh-hannover.de
Keywords Brain function - Brain death - Brain perfusion CT-angiography $\cdot$ Stasis filling $\cdot$ Quality control

\section{Einleitung}

Die neuen Richtlinien zur Feststellung des endgültigen, nicht behebbaren Ausfalls der Gesamtfunktion des Großhirns, des Kleinhirns und des Hirnstamms sind am 06.07.2015 im Deutschen Ärzteblatt veröffentlicht worden [1]. Diese nun vierte Richtlinienfortschreibung legt in Erfüllung des gesetzlichen Auftrags nach $\S 16$ Abs. 1 S. 1 Nr. 1 TPG (Transplantationsgesetz) den Stand der Erkenntnisse der medizinischen Wissenschaft für die Regeln zur Feststellung des Todes nach $\S 3$ Abs. 1 S. 1 Nr. 2 TPG und die Verfahrensregeln zur Feststellung des endgültigen, nicht behebbaren Ausfalls der Gesamtfunktion des Großhirns, des Kleinhirns und des Hirnstamms nach $\S 3$ Abs. 2 Nr. 2 TPG einschließlich der dazu jeweils erforderlichen ärztlichen Qualifikation fest. Der bisher verwendete Ausdruck „Hirntod“ wurde durch die medizinisch-wissenschaftlich präzisere Bezeichnung ,irreversibler Hirnfunktionsausfall“ ersetzt [1]. Eine wesentliche Neuerung besteht in der Zulassung der CTAngiographie als weiteres apparatives Verfahren zur Feststellung des zerebralen Zirkulationsstillstandes. Dieser ist häufig Ursache des irreversiblen Hirnfunktionsausfalls. Ist im Rahmen der Feststellung des endgültigen, nicht behebbaren Ausfalls der Gesamtfunktion des Großhirns, des Kleinhirns und des Hirnstamms der zerebrale Zirkulationsstillstand nachgewiesen worden, sind potentiell reversible Ursachen der klinischen Ausfallsymptome ausgeschlossen. Damit kann die Irreversibilität des Hirnfunktionsausfalls ohne Wartezeit und Verlaufsuntersuchung festgestellt werden [1]. Weitere zugelassene Verfahren sind die Doppler-/ Duplexsonographie und die Perfusionsszintigraphie. Die 
Indikationsstellung zur selektiven arteriellen Angiographie (DSA) erfordert wie bei den bisher gültigen Leitlinien [2] die Möglichkeit einer therapeutischen Konsequenz.

\section{Wie ist die CTA zur Feststellung des zerebralen Zirkulationsstillstandes einzusetzen und zu interpretieren?}

Die CT-Angiographie ist - wie in den neuen Richtlinien festgelegt - standardisiert durchzuführen (s. Protokoll in Tab. 1). Dies entspricht auch den Empfehlungen eines Cochrane Reviews zu diesem Thema [3]. Das CTA-Protokoll wurde mit dem Ziel der maximalen Sicherheit erstellt und basiert auf den Parametern der bisher größten deutschen Studie zum Einsatz der CTA zur Feststellung des zerebralen Zirkulationsstillstandes [4]. Die Bewertung erfolgt auch unter Berücksichtigung der Akzeptanz durch die anfordernden Ärzte und die Angehörigen der betroffenen Patienten sehr konservativ [1]. Die Beurteilung der CTund CTA-Aufnahmen muss durch Ärzte für Radiologie mit mehrjähriger neuroradiologischer Erfahrung, besser durch Radiologen mit dem „Schwerpunkt Neuroradiologie“ erfolgen. Bei exakter Umsetzung des Standardprotokolls kann diese Beurteilung auch von externen Spezialisten mit nachgewiesener Qualifikation durchgeführt werden.

Die Indikation zur CTA ist gerechtfertigt, wenn diese zur Klärung der Qualität der zerebralen Durchblutung durchgeführt wird. Wie bei jeder Gabe von Kontrastmitteln, sind die bekannten Vorsichtsmaßnahmen zum Schutze des Patienten einzuhalten.

Der zu prüfende zerebrale Zirkulationsstillstand tritt ein, wenn der intrakranielle Druck über dem arteriellen Mitteldruck liegt. In der Folge sistiert die metabolische Versorgung des Gehirns. Die Zuverlässigkeit der CT-Angiographie zur Erfassung des zerebralen Zirkulationsstillstandes wurde erstmals 1998 in einer größeren Studie von Dupas et al. [5] bestätigt. Auf diesen Ergebnissen basierend wurde die CTA zuerst in Frankreich, später in weiteren Ländern für die Diagnostik des irreversiblen Hirnfunktionsausfalles (international übliche Bezeichnung ,brain death“) zugelassen. Bei einer Cochrane-Analyse [3] dieser und folgender Studien [4, 6-13] wurde eine Sensitivität von $85 \%$ ermittelt, bei vorbestehendem Hirnfunktionsausfall einen zerebralen Zirkulationsstillstand nachzuweisen. Der fehlende Nachweis bei $15 \%$ der Patienten war im Wesentlichen auf traumatische oder iatrogene Eröffnungen der Kalotte zurückzuführen. Unter diesen Bedingungen kann ein Anstieg des intrakraniellen Druckes über den arteriellen Mitteldruck verhindert werden. War mittels CTA ein zerebraler Zirkulationsstillstand dokumentiert worden, wurde auch der endgültige, nicht behebbare Ausfall der Gesamtfunktion des Großhirns, des Kleinhirns und des Hirnstamms bestätigt.

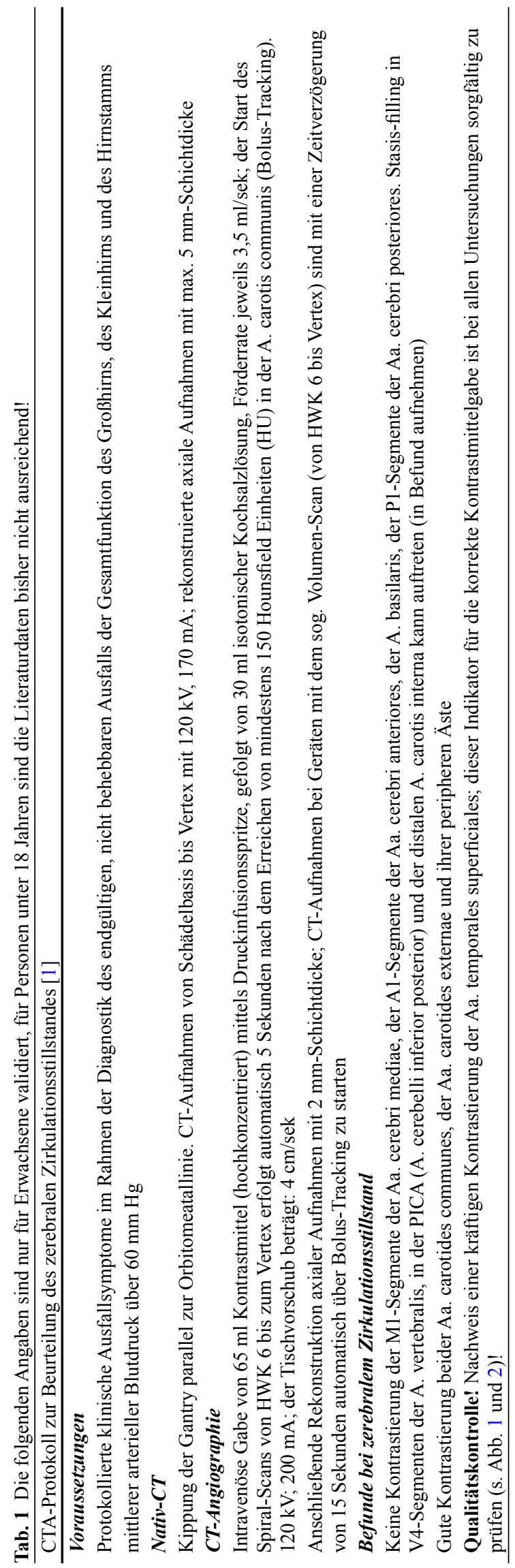



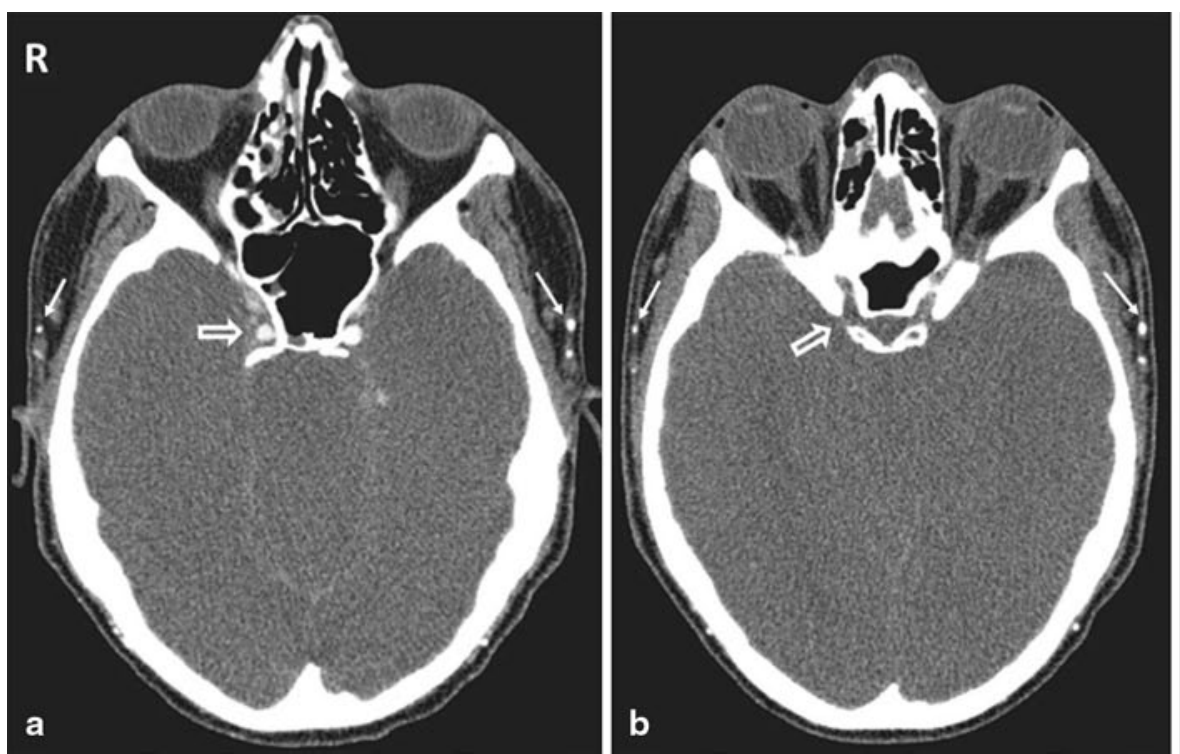

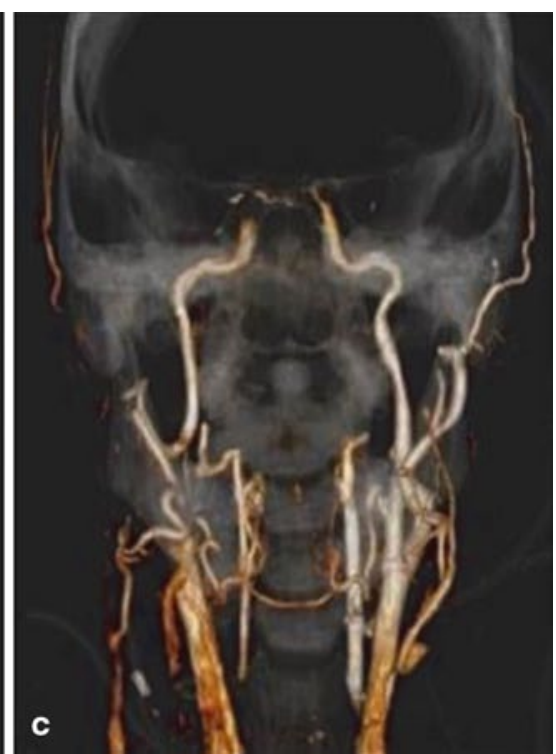

superficialis (kleine Pfeile (a) und (b); Qualitätskontrolle!). Die 3DDarstellung (c) zeigt den Abbruch der Kontrastierung in der distalen A. carotis interna bds. sowie der Vertebralarterien in Höhe HWK 2 vor Eintritt in das Foramen Magnum

Die erheblichen Erfolge der interventionellen Therapie des Schlaganfalls $[15,16]$ haben u. a. dazu beigetragen, dass die CT-Angiographie in Deutschland flächendeckend zur Dokumentation eines noch persistierenden Gefäßverschlusses eingesetzt wird. Im Gegensatz zum thrombotischen Verschluss beim Schlaganfall kommt es bei einem pathologischen Anstieg des intrakraniellen Druckes jedoch nicht zu einem Gefäßverschluss, sondern zu einem Druckbedingt verhinderten Einstrom von Blut in den intrakraniellen Raum. Mit zunehmendem zeitlichem Abstand von der intravenösen Kontrastmittelgabe kann das sog. ,stasis filling" auftreten als eine langsam progrediente Ausbreitung des Kontrastmittels in den intrakraniellen Arterien, zunächst intradural in den Aa. carotides internae und Aa. vertebrales sowie davon abgehenden intraduralen Arterien (PICA). Dies ist von einer zerebralen Durchblutung zu unterscheiden. Die Arbeitsgruppe von Sawicki et al. [17] hat diese zeitabhängige Kontrastmittelausbreitung intensiv mittels zusätzlicher Perfusionsmessungen untersucht. Als Ergebnis zeigte sich bei klinischen Zeichen des irreversiblen Hirnfunktionsausfalles und fehlender Kontrastierung der peripheren Großhirnarterien eine vollständig fehlende Perfusion, das heißt ein vollständiger zerebraler Zirkulationsstillstand. Bestätigt durch Studien von Welschehold und Mitarbeitern [18] sollte aufgrund des Stasis-Filling auf die zusätzliche Durchführung einer „venösen“ CTA verzichtet werden.

Entsprechend den Auswertekriterien des Standardprotokolls sollten zur Feststellung des zerebralen Zirkulationsstillstandes - bei guter Kontrastierung der A. carotis communis, der A. carotis externa und der A. vertebralis ausfalles dürfte jedoch auch bei Kindern nach dem ersten Lebensjahr (geschlossene Fontanellen) der Nachweis des zerebralen Zirkulationsstillstandes mittels CTA ein relevanter prognostischer Indikator sein 
Abb. 3 Obwohl eine ausgeprägte Hirnschwellung (a) besteht, ist noch eine residuale Kontrastierung der distalen A. basilaris, der A. cerebri posterior links und der tentoriellen Venen (Pfeile) nachzuweisen. Damit liegt nach den aktuellen Kriterien kein zerebraler Zirkulationsstillstand vor. Bei ausgeprägter basaler $\mathrm{SAB}$ (b, vor KM-Gabe) kann die Beurteilung eines zerebralen Zirkulationsstillstandes mittels CTA erschwert sein. Hier ist mit geeigneten Window- und Center-Einstellungen eine besonders sorgfältige Prüfung erforderlich
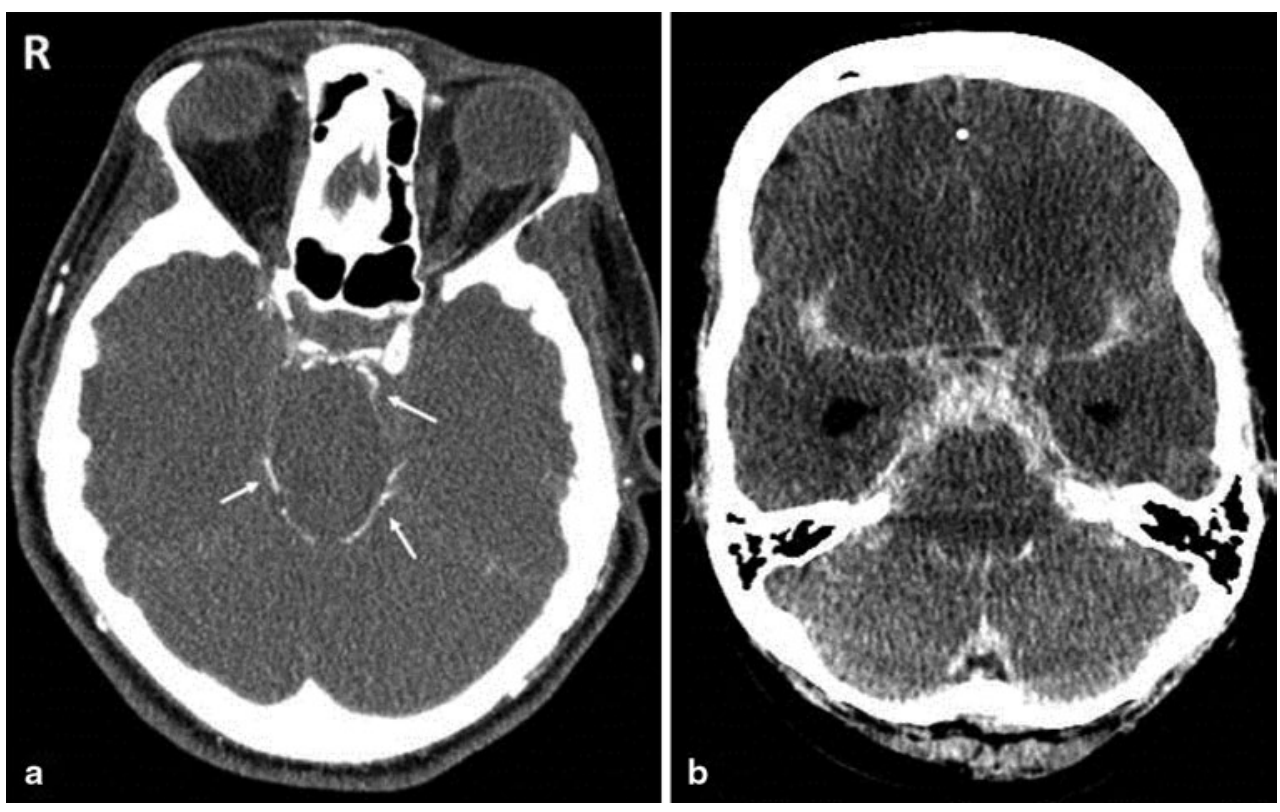

beidseits - keine Kontrastierungen der A1-Segmente der A. cerebri anterior, der M1-Segmente der A. cerebri media, der P1-Segmente der A. cerebri posterior beidseits oder der A. basilaris nachgewiesen werden. Sind Kontrastierungen in diesen Gefäßabschnitten erkennbar, ist der zerebrale Zirkulationsstillstand nach den aktuell gültigen Richtlinien nicht zweifelsfrei nachgewiesen (s. Abb. 3a). Dann ist eine weitere apparative Untersuchung (EEG, evozierte Potentiale, Doppler-/Duplexsonographie, Perfusionsszintigraphie, CTAngiographie) oder eine zweite klinische Verlaufsbeurteilung nach den vorgeschriebenen Wartezeiten durchzuführen [1]. Bei großen offenen Schädel-Hirn-Verletzungen und vereinzelt bei sekundären Hirnschädigungen kommt es, wenn der intrakranielle Druck nicht über den mittleren arteriellen Druck ansteigen kann, nicht zu einem zerebralen Zirkulationsstillstand. In diesen Fällen ist die Irreversibilität des Hirnfunktionsausfalls entweder durch klinische Verlaufsuntersuchungen nach den normierten Wartezeiten oder durch neurophysiologische Befunde nachzuweisen [1].

Open Access Dieser Artikel unterliegt den Bedingungen der Creative Commons Attribution License. Dadurch sind die Nutzung, Verteilung, und Reproduktion erlaubt, sofern der/die Originalautor/en und die Quelle angegeben sind.

\section{Literatur}

1. Beschluss der Bundesärztekammer über die. Richtlinie gemäß $\S 16$ Abs. 1 S. 1 Nr. 1 TPG für die Regeln zur Feststellung des Todes nach $\S 3$ Abs. 1 S. 1 Nr. 2 TPG und die Verfahrensregeln zur Feststellung des endgültigen, nicht behebbaren Ausfalls der Gesamtfunktion des Großhirns, des Kleinhirns und des Hirnstamms nach $\S 3$ Abs. 2 Nr. 2 TPG, Vierte Fortschreibung. Dtsch Arztebl. 2015; 112(27-28): A-1256/B-1052/C-1024.
2. Richtlinien zur Feststellung des Hirntodes. Dritte Fortschreibung 1997 mit Ergänzungen gemäß Transplantationsgesetz (TPG). Dtsch Arztebl. 1998;95(30):A 1861-8.

3. Taylor T, Dineen RA, Gardiner DC, Buss CH, Howatson A, Pace NL. Computed tomography (CT) angiography for confirmation of the clinical diagnosis of brain death. Cochrane Database Syst Rev. 2014;3:CD009694.

4. Welschehold S, Boor S, Reuland K, Thömke F, Kerz T, Reuland A, Beyer C, Gartenschläger M, Wagner W, Giese A, Müller-Forell W. Technical aids in the diagnosis of brain death-a comparison of SEP, AEP, EEG, TCD and CT angiography. Dtsch Arztebl Int. 2012;109:624-30.

5. Dupas B, Gayet-Delacroix M, Villers D, Antonioli D, Veccherini MF, Soulillou JP. Diagnosis of brain death using two-phase spiral CT. AJNR Am J Neuroradiol. 1998;19:641-7.

6. Berenguer CM, Davis FE, Howington JU. Brain death confirmation: comparison of computed tomographic angiography with nuclear medicine perfusion scan. J Trauma. 2010;68:553-9.

7. Bohatyrewicz R, Sawicki M, Walecka A, Walecki J, Rowinski O, Bohatyrewicz A, Kanski A, Czajkowski Z, Krzysztalowski A, Solek-Pastuszka J, Zukowski M, Marzec-Lewenstein E, Wojtaszek M. Computed tomographic angiography and perfusion in the diagnosis of brain death. Transplant Proc. 2010;42:3941-6.

8. Combes JC, Chomel A, Ricolfi F, d'Athis P, Freysz M. Reliability of computed tomographic angiography in the diagnosis of brain death. Transplant Proc. 2007;39:16-20.

9. Frampas E, Videcoq M, de Kerviler E, Ricolfi F, Kuoch V, Mourey F, Tenaillon A, Dupas B. CT angiography for brain death diagnosis. AJNR Am J Neuroradiol. 2009;30:1566-70.

10. Escudero D, Otero J, Marqués L, Parra D, Gonzalo JA, Albaiceta GM, Cofiño L, Blanco A, Vega P, Murias E, Meilan A, Roger RL, Taboada F. Diagnosing brain death by CT perfusion and multislice CT angiography. Neurocrit Care. 2009;11:261-71.

11. Leclerc X, Taschner CA, Vidal A, Strecker G, Savage J, Gauvrit JY, Pruvo JP. The role of spiral CT for the assessment of the intracranial circulation in suspected brain-death. J Neuroradiol. 2006;33:90-5.

12. Qureshi AI, Kirmani JF, Xavier AR, Siddiqui AM. Computed tomographic angiography for diagnosis of brain death. Neurology. 2004;62:652-3. 
13. Rieke A, Regli B, Mattle HP, Brekenfeld C, Gralla J, Schroth G, Ozdoba C. Computed tomography angiography (CTA) to prove circulatory arrest for the diagnosis of brain death in the context of organ transplantation. Swiss Med Wkly. 2011;141:w13261.

14. Welschehold S, Kerz T, Boor S, Reuland K, Thömke F, Reuland A, Beyer C, Wagner W, Müller-Forell W, Giese A. Detection of intracranial circulatory arrest in brain death using cranial CT-angiography. Eur J Neurol. 2013;20:173-9.

15. Berkhemer OA, Fransen PS, Beumer D, van den Berg LA, Lingsma HF, Yoo AJ, Schonewille WJ, Vos JA, Nederkoorn PJ, Wermer MJ, van Walderveen MA, Staals J, Hofmeijer J, van Oostayen JA, Lycklama à Nijeholt GJ, Boiten J, Brouwer PA, Emmer BJ, de Bruijn SF, van Dijk LC, Kappelle LJ, Lo RH, van Dijk EJ, de Vries J, de Kort PL, van Rooij WJ, van den Berg JS, van Hasselt BA, Aerden LA, Dallinga RJ, Visser MC, Bot JC, Vroomen PC, Eshghi O, Schreuder TH, Heijboer RJ, Keizer K, Tielbeek AV, den Hertog HM, Gerrits DG, van den Berg-Vos RM, Karas GB, Steyerberg EW, Flach HZ, Marquering HA, Sprengers ME, Jenniskens SF, Beenen LF, van den Berg R, Koudstaal PJ, van Zwam WH, Roos YB, van der Lugt A, van Oostenbrugge RJ, Majoie CB, Dippel DW; MR CLEAN Investigators. A randomized trial of intraarterial treatment for acute ischemic stroke. N Engl J Med. 2015;372:11-20.
16. Saver JL, Goyal M, Bonafe A, Diener HC, Levy EI, Pereira VM, Albers GW, Cognard C, Cohen DJ, Hacke W, Jansen O, Jovin TG, Mattle HP, Nogueira RG, Siddiqui AH, Yavagal DR, Baxter BW, Devlin TG, Lopes DK, Reddy VK, du Mesnil de Rochemont R, Singer OC, Jahan R; SWIFTPRIME Investigators. Stent-retriever thrombectomy after intravenous t-PA vs. t-PA alone in stroke. N Engl J Med. 2015;372:2285-95.

17. Sawicki M, Bohatyrewicz R, Safranow K, Walecka A, Walecki J, Rowinski O, Solek-Pastuszka J, Czajkowski Z, Marzec-Lewenstein E, Motyl K, Przybyl W, Czarnecka A. Dynamic evaluation of stasis filling phenomenon with computed tomography in diagnosis of brain death. Neuroradiology. 2013;55:1061-9.

18. Welschehold S, Kerz T, Boor S, Reuland K, Thömke F, Reuland A, Beyer C, Tschan C, Wagner W, Müller-Forell W, Giese A. Computed tomographic angiography as a useful adjunct in the diagnosis of brain death. J Trauma Acute Care Surg. 2013;74:1279-85. 